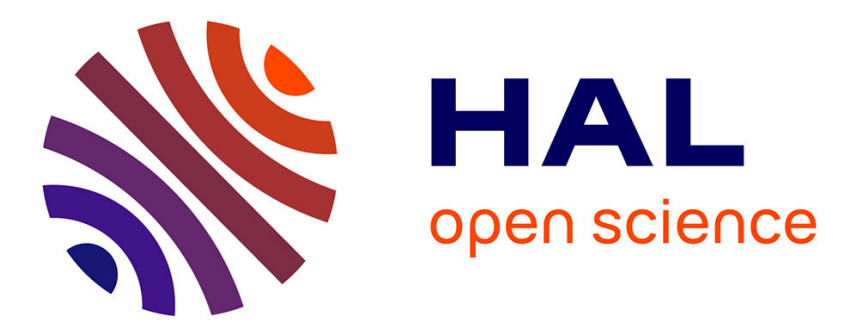

\title{
A constitutive model for fibrous tissues considering collagen fiber crimp
}

F. Cacho, P.J. Elbischger, J.F. Rodríguez, M. Doblaré, G.A. Holzapfel

\section{To cite this version:}

F. Cacho, P.J. Elbischger, J.F. Rodríguez, M. Doblaré, G.A. Holzapfel. A constitutive model for fibrous tissues considering collagen fiber crimp. International Journal of Non-Linear Mechanics, 2007, 42 (2), pp.391. 10.1016/j.ijnonlinmec.2007.02.002 . hal-00501746

\section{HAL Id: hal-00501746 https://hal.science/hal-00501746}

Submitted on 12 Jul 2010

HAL is a multi-disciplinary open access archive for the deposit and dissemination of scientific research documents, whether they are published or not. The documents may come from teaching and research institutions in France or abroad, or from public or private research centers.
L'archive ouverte pluridisciplinaire HAL, est destinée au dépôt et à la diffusion de documents scientifiques de niveau recherche, publiés ou non, émanant des établissements d'enseignement et de recherche français ou étrangers, des laboratoires publics ou privés. 


\title{
Author's Accepted Manuscript
}

A constitutive model for fibrous tissues considering collagen fiber crimp

F. Cacho, P.J. Elbischger, J.F. Rodríguez, M. Doblaré, G.A. Holzapfel

INTERNATIONAL JOURNAL OF

NON-LINEAR

MECHANICS

\author{
PII: $\quad$ S0020-7462(07)00055-8 \\ DOI: $\quad$ doi:10.1016/j.ijnonlinmec.2007.02.002 \\ Reference: $\quad$ NLM 1340
}

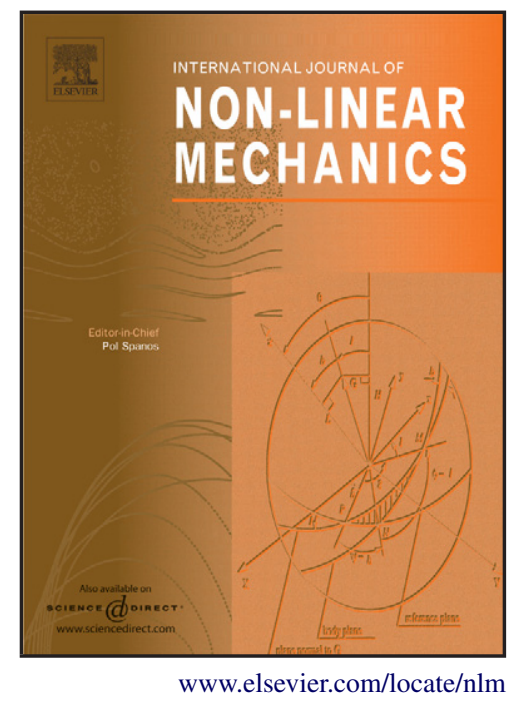

To appear in: International Journal of Non-

Linear Mechanics

Received date: 30 December 2006

Revised date: 6 February 2007

Accepted date: 6 February 2007

Cite this article as: F. Cacho, P.J. Elbischger, J.F. Rodríguez, M. Doblaré and G.A. Holzapfel, A constitutive model for fibrous tissues considering collagen fiber crimp, International Journal of Non-Linear Mechanics (2007), doi:10.1016/j.ijnonlinmec.2007.02.002

This is a PDF file of an unedited manuscript that has been accepted for publication. As a service to our customers we are providing this early version of the manuscript. The manuscript will undergo copyediting, typesetting, and review of the resulting galley proof before it is published in its final citable form. Please note that during the production process errors may be discovered which could affect the content, and all legal disclaimers that apply to the journal pertain. 


\title{
A constitutive model for fibrous tissues considering collagen fiber crimp
}

\author{
F. Cacho ${ }^{\mathrm{a}, \mathrm{b}}$, P.J. Elbischger ${ }^{\mathrm{c}}$, J.F. Rodríguez ${ }^{\mathrm{b}}$ \\ M. Doblaré ${ }^{b}$ and G.A. Holzapfel ${ }^{\mathrm{d}, \mathrm{e}}{ }^{\star}$ \\ anstitute for Structural Analysis, Computational Biomechanics \\ Graz University of Technology, Austria \\ ${ }^{\mathrm{b}}$ Group of Structural Mechanics and Materials Modeling \\ Aragon Institute of Engineering Research (I3A), University of Zaragoza, Spain \\ ${ }^{\mathrm{c}}$ Medical Information Technology, Carinthia Tech Institute \\ University of Applied Sciences, Klagenfurt, Austria \\ dDepartment of Solid Mechanics, School of Engineering Sciences \\ Royal Institute of Technology (KTH), Stockholm, Sweden \\ eInstitute for Biomechanics, Graz University of Technology, Austria
}

\begin{abstract}
A micromechanically-based constitutive model for fibrous tissues is presented. The model considers the randomly crimped morphology of individual collagen fibers, a morphology typically seen in photomicrographs of tissue samples. It describes the relationship between the fiber endpoints and its arc-length in terms of a measurable quantity, which can be estimated from image data. The collective mechanical behavior of collagen fibers is presented in terms of an explicit expression for the strain-energy function, where a fiber-specific random variable is approximated by a Beta distribution. The model-related stress and elasticity tensors are provided. Two representative numerical examples are analyzed with the aim of demonstrating the peculiar mechanism of the constitutive model and quantifying the effect of parameter changes on the mechanical response. In particular, a fibrous tissue, assumed to be (nearly) incompressible, is subject to a uniaxial extension along the fiber direction, and, separately, to pure shear. It is shown that the fiber crimp model can reproduce several of the expected characteristics of fibrous tissues.
\end{abstract}

Key words: Collagen fiber, constitutive model, fiber crimp, micromechanics, statistical distribution.

\footnotetext{
* Corresponding author. Institute for Biomechanics, Center for Biomedical Engineering, Graz University of Technology, Kronesgasse 5, 8010 Graz, Austria. E-mail address: gh@biomech.tu-graz.ac.at (G.A. Holzapfel).
} 


\section{Introduction}

The central role of collagen as the major structural protein of mammalian tissue, comprising approximately one-third of the total protein in mammalian organisms, has motivated a significant effort towards determining its mechanical properties at all levels, ranging from single monomers [1,2] and long-chain polymers $[3,4]$ to a structural element within a (macroscopic) biological tissue [5-8].

On the basis of the mechanical properties, a number of constitutive models have been developed in the past in attempts to describe the experimental data. While at the microscopic level, chain models such as the (Kratky-Porod) worm-like model are popular [9-11], at the macroscopic level the continuum theory of finite elastic deformations of solids reinforced with fibers is frequently the constitutive theory of choice. The basic ideas of the theory are contained in [12], with further developments on strongly anisotropic solids in [13], and applications to model, e.g., arterial walls in $[14,15]$; see also the recent volume [16]. In such macroscopic models the collagen fibers are assumed to be continuously arranged in the matrix material, as utilized in [17], and the characteristic nonlinear stiffening is best represented by means of an exponential function. Effective alternatives are based on limiting chain models, see, for example, [18], and references therein.

The pioneering work by Lanir $[19,20]$ on the mechanics of fibrous (connective) tissues as a consequence of its microstructure has influenced much of the works on microstructural constitutive models. Essentially, the works $[19,20]$ postulate that the fibers are crimped and that they have different lengths so that for a given macroscopic deformation in the material each individual fiber is stretched differently. There is, thus, a distribution in either the stretch of the fibers or their lengths. This idea has also been adopted subsequently by means of constitutive models to describe the mechanical response of, e.g., arterial walls ([21] with ideas from [22,14]) or tendons and ligaments [23], just to name a few. All these constitutive models, however, assume unbounded statistical distributions for the fiber length (or stretch), which is a bounded quantity. In addition, in these models no attempt has been made to correlate the fiber morphology (crimp) with the associated mechanical response in the form of stress-stretch relationships. It was Lanir who considered the possibility that the stretch could be nonuniform due to crimping, with a generic distribution along the fiber axis, which he assumed to be Gaussian. Recently, Freed and Doehring [24] have proposed a model where crimped fibrils in a fascicle are approximated as a helical spring. Thereby, the collagen fiber waveforms have a pre-defined arrangement; no statistical distribution is used. In different works, such as [25-27], the distribution of the fiber orientations has been addressed; however, therein, the mechanical properties of the collagen fibers 
within the tissue were considered to be independent of the degree of crimping.

In this paper a new constitutive model for the macroscopic behavior of fibrous tissues is presented. It takes the randomly crimped morphology of the individual collagen fibers into account. In Section 2 a statistical description of the fiber crimp is developed, which is used in Section 3 to model the collective behavior of fibers. In Section 4 the mechanical behavior of a fibrous tissue, assumed to be (nearly) incompressible, is analyzed in detail. The tissue is subject to a stretch-controlled uniaxial extension along the fiber direction, and, separately, to pure shear. In particular, the effect of the different model parameters on the mechanical response is studied. The final section contains a brief discussion together with a description of some limitations of the proposed constitutive model.

\section{Statistical and constitutive description of a single collagen fiber}

In unloaded tissue samples collagen fibers show a wavy structure. In this section we develop a model that incorporates the random crimp of collagen fibers to be characterized.

\subsection{Random crimp of a single fiber}

We start by considering a set of randomly generated data in an interval of length $L_{0}+w$ on the $X$-axis such that at any point $x$ within that interval the associated coordinates $y$ and $z$ are independent and normally distributed random variables with zero mean. Under this condition the data generated can be regarded as white Gaussian noise, and characterized by the variance $\sigma^{2}$. In the following it is assumed that the variances in the $Y$ and $Z$-directions are equal, in other words the fiber undulates with equal characteristics in all directions orthogonal to the $X$-axis.

The randomness of the data generated may be larger than that of an actual fiber. By applying a smoothing function or filter $h$, which averages the coordinates of the points in a neighborhood $[-w / 2, w / 2]$ of each point, a derived set of data in the interval $\left[0, L_{0}\right]$ is obtained. It is implicitly assumed that $h$ and its first derivative has compact support in $[-w / 2, w / 2]$. The resulting data are also random and normally distributed with zero mean since the filtering operation does not affect the Gaussian nature of the distribution. As a consequence, the variance of the new random variable is unequivocally related to that of the white Gaussian noise through the filter. 
(a)

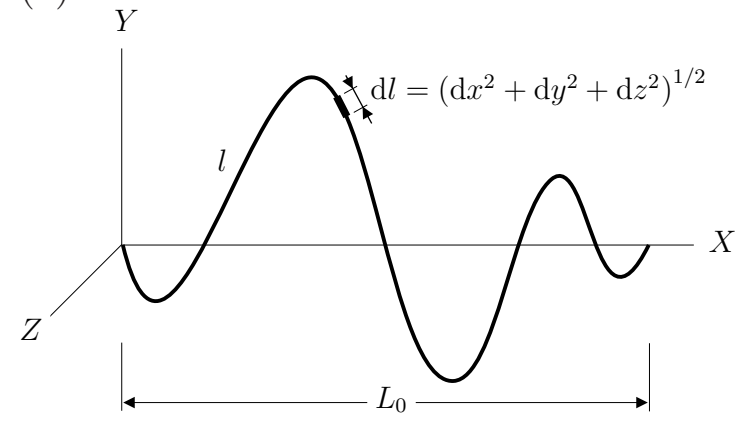

(b)

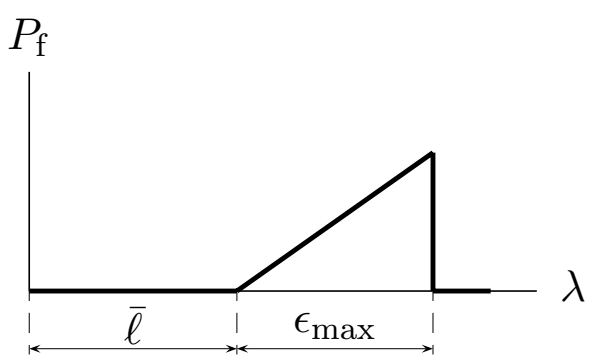

Fig. 1. (a) Schematic representation of a single fiber; (b) stress-stretch behavior of a single fiber (see eq. (6)).

The infinitesimal arc-length $\mathrm{d} l$ of the fiber is then (see Fig. 1(a))

$$
\mathrm{d} l=\left(\mathrm{d} x^{2}+\mathrm{d} y^{2}+\mathrm{d} z^{2}\right)^{1 / 2}
$$

where $\mathrm{d} y$ and $\mathrm{d} z$ are related to $\mathrm{d} x$ through the derivative of the filter. Thus, we can write $d=\mathrm{d} y / \mathrm{d} x=\mathrm{d} z / \mathrm{d} x$, where $d$ is a zero mean, normally distributed random variable whose variance $\sigma_{d}^{2}$ can be directly related to $\sigma^{2}$. Therefore, it follows that

$$
\mathrm{d} l=\sqrt{2 d^{2}+1} \mathrm{~d} x=\ell \mathrm{d} x,
$$

where $\ell$ is a random variable, which is neither zero mean nor normally distributed, and whose probability density function, subsequently abbreviated as $\mathcal{P}$, is (see Appendix A.1)

$$
\mathcal{P}=\frac{\ell}{\sigma_{d}^{2}} \exp \left(-\frac{\ell^{2}-1}{2 \sigma_{d}^{2}}\right), \quad \ell \geq 1 .
$$

Relation (2) describes the arc-length at infinitesimal scale within a single fiber. Our interest is, however, the establishment of (2) at the fiber level, i.e.

$$
l=\int_{x=0}^{L_{0}} \ell \mathrm{d} x \approx \bar{\ell} L_{0}
$$

where $\bar{\ell}$ is a fiber-specific quantity that denotes the mean (or expected value) of $\ell$, i.e. (see Appendix A.2)

$$
\bar{\ell}=\sqrt{2 \sigma_{d}^{2} \exp \left(1 / \sigma_{d}^{2}\right)} \Gamma\left(\frac{3}{2}, \frac{1}{2 \sigma_{d}^{2}}\right),
$$

where $\Gamma(\bullet, \bullet)$ is the upper incomplete Gamma function [28]. As $L_{0}$ increases with respect to the fiber wavelength, approximation $(4)_{2}$ improves so that we 

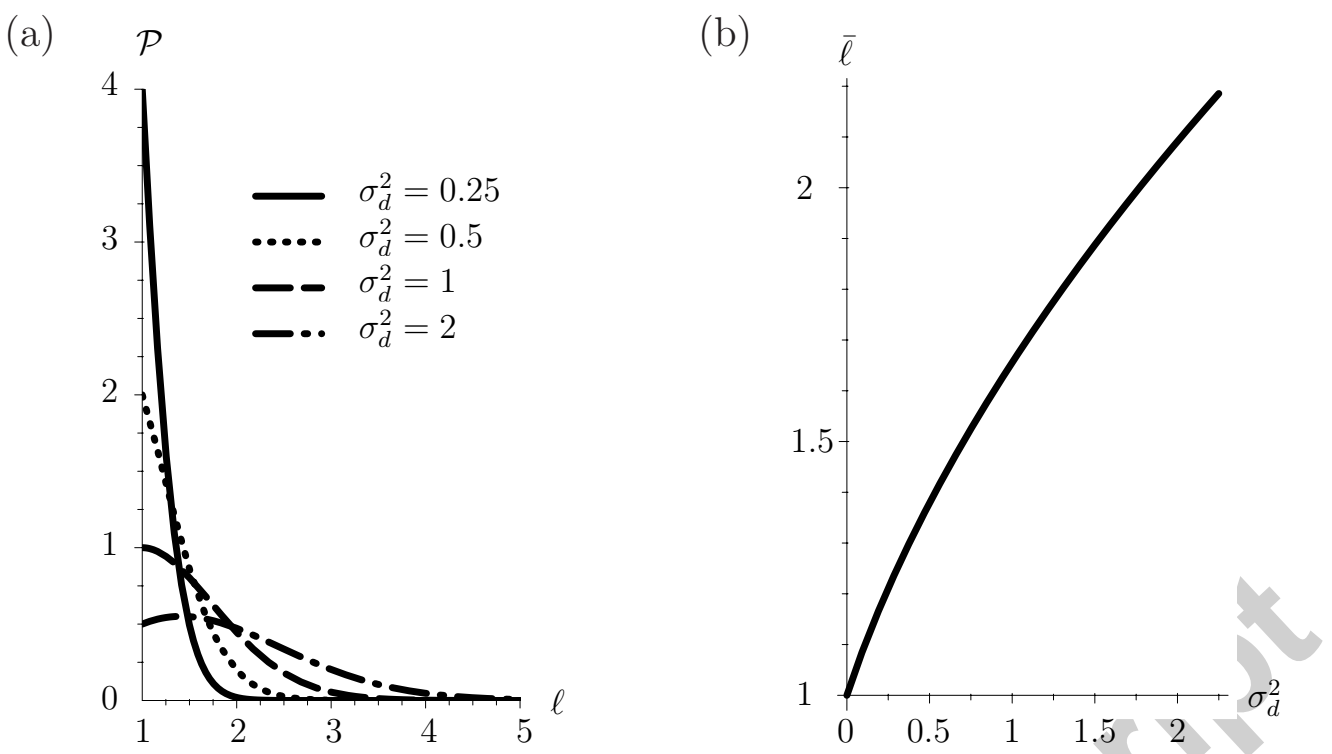

Fig. 2. (a) $\mathcal{P}$ versus $\ell$ for different values of $\sigma_{d}^{2}$ according to (3); (b) dependence of $\bar{\ell}$ on $\sigma_{d}^{2}$ according to (5).

can assume that the linear relationship $l=\bar{\ell} L_{0}$ holds for (very) long fibers like those in fibrous tissues (see, e.g., [29]).

At this point it is important to note that the probability density function $\mathcal{P}$ and the mean $\bar{\ell}$ depend only on the variance $\sigma_{d}^{2}$, which is a (measurable) quantity that can be obtained from the fiber morphology typically seen in micrographs [30]. This quantity is determined by the frequency content of the fiber. The question therefore arises as to what the range of $\sigma_{d}^{2}$ should be since it is clear that the higher its value the more is the waviness. A comparison of computer-generated curves with typical fibers, as can be seen in images taken through a microscope of, e.g., the outermost artery layer, motivates a restriction of the values of $\sigma_{d}^{2}$ to the interval [0,2.25]. Curves that are generated within this interval look very similar to those seen in micrographs of real samples [30].

The dependence of $\mathcal{P}$ and $\bar{\ell}$ on $\sigma_{d}^{2}$ has been plotted in Fig. 2.

\subsection{Constitutive model of a single fiber}

The long and thin collagen fibers are essentially one-dimensional entities. Their wavy appearance in the reference configuration motivates the assumption that they are unable to sustain compressive loads. For the modeling of the tensile behavior, we follow the work of others and consider that a given fiber carries load only after unfolding, assuming that the force necessary to perform this 
is negligible $[22,23]$. Once the fiber starts to bear load, it is assumed that it follows Hooke's law until rupture [31].

The parameter $\bar{\ell}$, thus, enters the constitutive model as the stretch at which the fiber becomes mechanically active, and, subsequently, it will be called (zero force) 'stretchability'. In terms of the engineering stress $P_{\mathrm{f}}$ in a single collagen fiber, the constitutive model may be written piece-wise as

$$
P_{\mathrm{f}}= \begin{cases}0 & \lambda \leq \bar{\ell} \\ k(\lambda-\bar{\ell}) & \bar{\ell}<\lambda \leq \bar{\ell}+\epsilon_{\max } \\ 0 & \lambda>\bar{\ell}+\epsilon_{\max }\end{cases}
$$

where the subindex $\mathrm{f}$ emphasizes the fact that this relationship applies to a single fiber, $k$ denotes the elastic modulus of the fiber, $\lambda$ is the fiber stretch, and $\epsilon_{\max }$ is the maximum stretch the fiber can undergo after unfolding (before failure). For an illustration of (6) see Fig. 1(b). Note that, while $\bar{\ell}$ is a fiber-specific (geometric) quantity, $\epsilon_{\max }$ and $k$ may be considered as material constants with physical meaning. The symbol $\epsilon$ is used here to denote finite (unidimensional) stretch.

As $P_{\mathrm{f}}$ and $\lambda$ are work conjugate, the associated elastic strain energy $\psi_{\mathrm{f}}$ stored in a single fiber with stretch $\lambda$ is

$$
\psi_{\mathrm{f}}(\lambda, \bar{\ell})=\frac{k}{2}(\lambda-\bar{\ell})^{2}, \quad \bar{\ell}<\lambda \leq \bar{\ell}+\epsilon_{\max }
$$

Hence, the energy needed to rupture a fiber equals the elastic energy stored at maximum stretch, i.e.

$$
\psi_{\max }=\frac{k}{2} \epsilon_{\max }^{2},
$$

which is independent of the fiber-specific (geometric) morphology.

\section{Mechanical behavior of fibrous tissues}

\subsection{General mechanical behavior}

The higher-order structure of fibrous tissues is formed by fiber bundles, which are groups of fibers that share a common orientation, subsequently called fiber families. Different fiber families may have different orientations such as is the case in the outermost layer (the adventitia) of a human artery. In the following developments we neglect the statistical distribution of fiber orientations and consider that within a fiber family the fibers have the same mean orientation. We may describe the macroscopic mechanical behavior of a collagen fiber 
family by means of a continuum formulation, which also simplifies numerical implementations.

We consider a representative volume element within the fibrous tissue and assume that the fibers and the matrix material do not interact mechanically, but undergo affine deformations described by the right Cauchy-Green tensor C. Under these conditions, the strain energy $\psi_{\text {tissue }}$ stored in the tissue may be written as

$$
\psi_{\text {tissue }}=\psi_{\text {matrix }}(\mathbf{C})+\sum_{i=1}^{N} \psi_{\text {fibers }}\left(\mathbf{C}, \mathbf{A}_{i}\right),
$$

applying an additive split, as first proposed in [32]. This equation considers $N$ distinct fiber families with specific orientations, each of which is described by means of a unit vector $\mathbf{a}_{i_{0}}$ in the reference configuration, with the resulting dyadic product $\mathbf{A}_{i}=\mathbf{a}_{i_{0}} \otimes \mathbf{a}_{i_{0}}$. The quantity $\mathbf{A}_{i}$ denotes the structure tensor associated with family $i$ so that the corresponding stretch $\lambda_{i}$ of the fiber family $i$ is

$$
\lambda_{i}=\left(\mathbf{C}: \mathbf{A}_{i}\right)^{1 / 2} .
$$

In a general situation, $\mathbf{a}_{i_{0}}$ is a three-dimensional vector. Note that according to the continuum theory of finite elastic deformations of solids reinforced with fibers, the fibers are assumed to be infinitesimally thin [12].

In eq. (9), the term $\psi_{\text {matrix }}$ represents the behavior of the aqueous, gel-like matrix material in which the fiber families are embedded. This substance is usually considered to behave isotropically. Although several models capable of describing large deformations are suitable for $\psi_{\text {matrix }}$ [33], it is common to apply the neo-Hookean model because of its simplicity (see, e.g., [17, Chapter $6]$ ). For the fibers, we assume that the morphology is independent of the orientation. It is clear, however, that each fiber can have a different waviness due to the intrinsic randomness of the fiber assembly process. This leads to the realization that the stretchability $\bar{\ell}$ is a statistically distributed variable in the tissue (across the fibers), with distribution $\mathcal{P}(\bar{\ell})$.

In the subsequent development, without loss of generality, we consider a fibrous tissue with only one family of collagen fibers $(N=1)$. At a given stretch $\lambda$ some fibers in the tissue may still be slack, if $\bar{\ell}$ is greater than the stretch $\lambda$ (see Fig. 1(b)). Therefore, only those fibers that have been already unfolded can contribute to $\psi_{\text {fibers }}$ according to

$$
\psi_{\text {fibers }}=\int_{\bar{\ell}=1}^{\lambda} \psi_{\mathrm{f}}(\lambda, \bar{\ell}) \mathcal{P}(\bar{\ell}) \mathrm{d} \bar{\ell} .
$$

Subsequently, we use the symbol $\psi$ instead of $\psi_{\text {fibers }}$ for simplicity.

According to (5), $\bar{\ell}$ depends nonlinearly on $\sigma_{d}^{2}$, which has a limited range, as discussed in Section 2.1. Numerical tests suggest that $\mathcal{P}(\bar{\ell})$ can be approxi- 

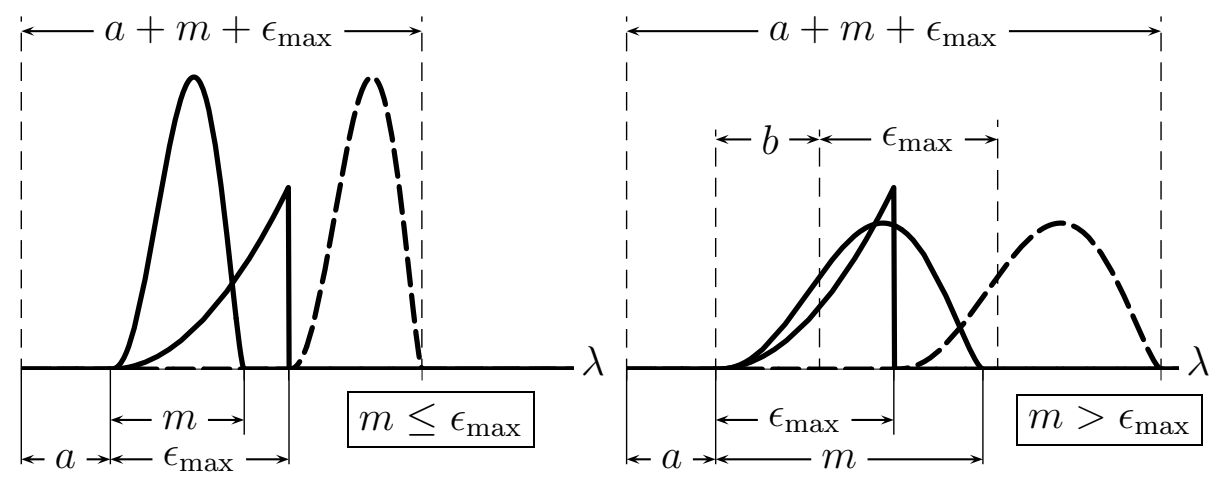

Fig. 3. Schematic representation of two possible loading phases distinguished by $m$, the range of the fiber-specific quantity $\bar{\ell}: m \leq \epsilon_{\max }$ in which fully recruited fibers are reached before tissue failure, and $m>\epsilon_{\max }$ in which the fibers start to fail before the maximum stretch is reached. The bell-shaped curves show the Beta distribution (both sketches are based on the same parameters, only $m$ is different), while the other nonlinear curves represent the stiffening of the tissue up to failure. The parameters $a$ and $\epsilon_{\max }$ denote the value of the stretchability $\bar{\ell}$ at minimum $\sigma_{d}^{2}$, and the maximum stretch a fiber can undergo after unfolding, respectively. The parameter $b$ refers to the unloading/reloading behavior (see Section 3.3).

mated satisfactorily by the Beta function, which has lower and upper limits and can represent symmetric and non-symmetric datasets [34]. In what follows, the lower limit is denoted by $a$, i.e. the value of $\bar{\ell}$ when $\sigma_{d}^{2}$ takes on its smallest value, while the upper limit is denoted by $a+m$, i.e. the value of $\bar{\ell}$ at which $\sigma_{d}^{2}$ is a maximum. Hence, $m$ is the range of $\bar{\ell}$ describing the fiber morphology across the tissue. According to [28] the (symmetric) Beta function is

$$
\beta(\eta, \gamma)=\frac{\Gamma(\eta) \Gamma(\gamma)}{\Gamma(\eta+\gamma)}
$$

where $\eta$ and $\gamma$ are called shape parameters, and $\Gamma(\bullet)$ denotes the Gamma function with argument $\bullet$.

\subsection{Tissue behavior during monotonic loading}

For subsequent developments it is convenient to introduce the modified parameters $\bar{\lambda}=\lambda-a$ and $\tilde{\ell}=\bar{\ell}-a$, where $\tilde{\ell}$ is then within the range $[0, m]$. With these variable changes and the introduction of $a$ as the lower limit, eq. (11) can be rewritten as

$$
\psi=\int_{\tilde{\ell}=0}^{\bar{\lambda}} \psi_{\mathrm{f}}(\lambda, \tilde{\ell}) \beta_{0}^{m}(\eta, \gamma ; \tilde{\ell}) \mathrm{d} \tilde{\ell}
$$


where

$$
\beta_{0}^{m}(\eta, \gamma ; \tilde{\ell})=\frac{1}{m} \frac{1}{\beta(\eta, \gamma)}\left(\frac{\tilde{\ell}}{m}\right)^{\gamma-1}\left(1-\frac{\tilde{\ell}}{m}\right)^{\eta-1}
$$

represents the two-parameter Beta distribution of $\tilde{\ell} \in[0, m]$. Consequently, by introducing the hypergeometric function ${ }_{2} F_{1},[28]$, the integral in (13) can be written as

$\psi=2 B\left(\frac{\bar{\lambda}}{m}\right)_{2}^{\gamma+2} F_{1}\left(1-\eta, \gamma, \gamma+3, \frac{\bar{\lambda}}{m}\right), \quad B=\frac{k}{2} \frac{1}{\beta(\eta, \gamma)} \frac{m^{2}}{\gamma(\gamma+1)(\gamma+2)}$

with $0 \leq \bar{\lambda} \leq \min \left(m, \epsilon_{\max }\right)$, and $\beta$ given by (12). Note that, although the behavior of a single fiber is considered to be linear, the tissue as a whole behaves nonlinearly, as described in (15).

When a fibrous tissue is subject to tensile loading in the direction of the fiber orientation, we then distinguish between two cases for the proposed model (see also Fig. 3):

(i) $m \leq \epsilon_{\max }$ : in this case the fibers are gradually recruited, as the tissue is stretched. At a certain point, all fibers are then unfolded and the stiffness due to the fibers is constant. Hence, a fully recruited elastic phase is reached before tissue failure.

(ii) $m>\epsilon_{\max }$ : in this case the fibers start to fail when the maximum stretch $\epsilon_{\max }$ is reached, while other fibers are still slack. This is a phase we call 'mixed recruitment/failure'.

For case (i), eq. (15) can be simplified to

$$
\psi=\frac{k}{2}\left[\bar{\lambda}^{2}-\frac{2 m \gamma \bar{\lambda}}{\eta+\gamma}+\frac{\gamma(\gamma+1) m^{2}}{(\eta+\gamma)(\eta+\gamma+1)}\right], \quad m<\bar{\lambda} \leq \epsilon_{\max }
$$

while, for case (ii), integration of (13) gives the strain energy

$$
\psi=2 B\left(\frac{\bar{\lambda}}{m}\right)_{2}^{\gamma+2} F_{1}\left(1-\eta, \gamma, \gamma+3, \frac{\bar{\lambda}}{m}\right)+\hat{\psi}, \quad \epsilon_{\max }<\bar{\lambda} \leq m
$$

where the notations 


$$
\begin{aligned}
\hat{\psi}= & -B\left(\frac{\hat{\lambda}}{m}\right)^{\gamma+1} \frac{\bar{\lambda}+\epsilon_{\max }}{m}(\gamma+1)(\gamma+2)_{2} F_{1}\left(1-\eta, \gamma, \gamma+1, \frac{\hat{\lambda}}{m}\right) \\
& +2 B\left(\frac{\hat{\lambda}}{m}\right)^{\gamma+1} \frac{\bar{\lambda}}{m} \gamma(\gamma+2)_{2} F_{1}\left(1-\eta, \gamma+1, \gamma+2, \frac{\hat{\lambda}}{m}\right) \\
& -B\left(\frac{\hat{\lambda}}{m}\right)^{\gamma+2} \gamma(\gamma+1)_{2} F_{1}\left(1-\eta, \gamma+2, \gamma+3, \frac{\hat{\lambda}}{m}\right),
\end{aligned}
$$

and $\hat{\lambda}=\bar{\lambda}-\epsilon_{\max }$ have been used.

If $\bar{\lambda}$ increases beyond $\max \left(m, \epsilon_{\max }\right)$ (beyond either full recruitment or mixed recruitment/failure) the strain energy required to stretch the tissue until complete failure (i.e. at $\bar{\lambda}=m+\epsilon_{\max }$ ) is

$\psi=\frac{k}{2}\left[\bar{\lambda}^{2}-\frac{2 m \gamma \bar{\lambda}}{\eta+\gamma}+\frac{\gamma(\gamma+1) m^{2}}{(\eta+\gamma)(\eta+\gamma+1)}\right]+\hat{\psi}, \quad \max \left(m, \epsilon_{\max }\right)<\bar{\lambda} \leq m+\epsilon_{\max }$

Equations (15)-(19) define the various energies required to stretch the (fibrous part of the) tissue monotonically along the fiber orientation. For $m \leq \epsilon_{\max }$ we then have

$$
\psi= \begin{cases}\text { eq. (15) } & 0 \leq \bar{\lambda} \leq m \\ \text { eq. (16) } & m<\bar{\lambda} \leq \epsilon_{\max } \\ \text { eq. (19) } & \epsilon_{\max }<\bar{\lambda} \leq m+\epsilon_{\max }\end{cases}
$$

while the equations summarize for $m>\epsilon_{\max }$ according to

$$
\psi= \begin{cases}\text { eq. (15) } & 0 \leq \bar{\lambda} \leq \epsilon_{\max } \\ \text { eq. (17) } & \epsilon_{\max }<\bar{\lambda} \leq m \\ \text { eq. (19) } & m<\bar{\lambda} \leq m+\epsilon_{\max }\end{cases}
$$

Note that the first and third rows in (20) and (21) involve the same equations, however, the range of $\bar{\lambda}$ is different; depending on the relative values of $m$ and $\epsilon_{\max }$.

\subsection{Tissue behavior during unloading/reloading}

We assume that reloading follows the last unloading path, and neglect viscous effects. Similarly to Section 3.2, for the unloading/reloading process we may also distinguish two cases: (i) elastic unloading/reloading, where the fibers are unfolded (gradually recruited) as before; (ii) tissue damage occurs due 
to individual fiber failure upon further stretching (mixed recruitment/failure phase).

The energy required for elastic unloading/reloading is

$$
\psi=2 B\left(\frac{\bar{\lambda}}{m}\right)_{2}^{\gamma+2} F_{1}\left(1-\eta, \gamma, \gamma+3, \frac{\bar{\lambda}}{m}\right)+\psi_{b}, \quad b \leq \bar{\lambda} \leq \min \left(m, \epsilon_{\max }\right)
$$

Here the notations

$$
\begin{aligned}
\psi_{b}= & -B \frac{\bar{\lambda}^{2}}{m^{2}}\left(\frac{b}{m}\right)^{\gamma}(\gamma+1)(\gamma+2)_{2} F_{1}\left(1-\eta, \gamma, \gamma+1, \frac{b}{m}\right) \\
& +2 B \frac{\bar{\lambda}}{m}\left(\frac{b}{m}\right)^{\gamma+1} \gamma(\gamma+2)_{2} F_{1}\left(1-\eta, \gamma+1, \gamma+2, \frac{b}{m}\right) \\
& -B\left(\frac{b}{m}\right)^{\gamma+2} \gamma(\gamma+1)_{2} F_{1}\left(1-\eta, \gamma+2, \gamma+3, \frac{b}{m}\right)
\end{aligned}
$$

and $b=\lambda_{\max }-a-\epsilon_{\max }$ (see Fig. 3) have been used, where $\lambda_{\max }$ denotes the maximum stretch reached during the loading history.

For $m \leq \epsilon_{\max }$ all non-failed fibers can be stretched in the unloading/reloading path, and the above expression turns into

$$
\psi=\frac{k}{2}\left[\bar{\lambda}^{2}-\frac{2 m \gamma \bar{\lambda}}{\eta+\gamma}+\frac{\gamma(\gamma+1) m^{2}}{(\eta+\gamma)(\eta+\gamma+1)}\right]+\psi_{b}, \quad m<\bar{\lambda} \leq b+\epsilon_{\max }
$$

The expressions (20)-(24) can now be reconciled, and written for general loading conditions as

$$
\psi= \begin{cases}\text { if } \bar{\lambda}=\bar{\lambda}_{\max } \text { and } \delta \bar{\lambda} \geq 0 & \text { eq. }(20) \text { for } m \leq \epsilon_{\max }, \\ \text { else if } b \leq \bar{\lambda} \leq \min \left(m, \epsilon_{\max }\right) & \text { eq. }(21) \text { for } m>\epsilon_{\max }, \\ \text { else if } m<\bar{\lambda} \leq b+\epsilon_{\max } & \text { eq. }(22), \\ \text { else if } b<\bar{\lambda} & 0,\end{cases}
$$

where $\delta \bar{\lambda}$ represents an admissible variation of $\bar{\lambda}$. 
Table 1

\begin{tabular}{l|ccc} 
Parameter & Symbol & Value & Dimension \\
\hline Elastic modulus of the fiber & $k$ & 400 & $\mathrm{MPa}$ \\
Maximum fiber stretch & $\epsilon_{\max }$ & 0.2 & - \\
Shape parameters & $\eta$ & 4.5 & - \\
& $\gamma$ & 2.8 & - \\
Range of $\bar{\ell}$ & $m$ & $0.15,0.25$ & - \\
Range of damaged fibers & $b$ & 0.05 & - \\
\hline
\end{tabular}

Parameter values used for the numerical examples.

\section{Numerical examples}

In this section we provide the stress and elasticity tensors for the considered constitutive model proposed in Section 3 and two representative numerical examples that demonstrate the applicability of the model. The parameters used for the constitutive model are chosen arbitrarily, and are summarized in Table 1.

\subsection{Stress and elasticity tensors}

For both examples the fibrous tissue is assumed to be (nearly) incompressible. The mechanical behavior of the matrix material is assumed to follow the neoHookean model. Thus,

$$
\psi_{\text {matrix }}=\frac{c}{2}\left(I_{1}-3\right), \quad I_{1}=\mathbf{C}: \mathbf{I}
$$

where $\mathbf{I}$ is the identity tensor. The stresses are derived from the strain-energy function according to, [17],

$$
\mathbf{S}_{\text {tissue }}=-J p \mathbf{C}^{-1}+2 \frac{\partial \psi_{\text {tissue }}}{\partial \mathbf{C}}=-J p \mathbf{C}^{-1}+\mathbf{S}_{\text {matrix }}+\mathbf{S}_{\text {fibers }},
$$

where $J=(\operatorname{det} \mathbf{C})^{1 / 2}$ denotes the volume ratio, with $J=1$ for the incompressible limit, and $p$ is the hydrostatic pressure. The tensor $\mathbf{S}_{\text {tissue }}$ is the second Piola-Kirchhoff stress tensor, and $\mathbf{S}_{\text {matrix }}$ and $\mathbf{S}_{\text {fibers }}$ are the contributions to it from the matrix and fibers, respectively. By means of eqs. (26), (10) and the chain rule, we find the explicit expressions

$$
\mathbf{S}_{\text {matrix }}=2 \frac{\partial \psi_{\text {matrix }}(\mathbf{C})}{\partial \mathbf{C}}=c \mathbf{I},
$$




$$
\mathbf{S}_{\text {fibers }}=2 \sum_{i=1}^{N} \frac{\partial \psi_{\text {fibers }}\left(\mathbf{C}, \mathbf{A}_{i}\right)}{\partial \mathbf{C}}=\sum_{i=1}^{N} \frac{1}{\lambda_{i}} \psi_{\lambda_{i}} \mathbf{A}_{i},
$$

where the abbreviation $\psi_{\lambda_{i}}=\partial \psi_{\text {fibers }}\left(\lambda_{i}\right) / \partial \lambda_{i}$ has been introduced, with the individual strain energies $\psi_{\text {fibers }}$ summarized in (25). Note that $\partial \psi_{\text {fibers }} / \partial \lambda_{i}=$ $\partial \psi_{\text {fibers }} / \partial \bar{\lambda}_{i}$, as $\lambda_{i}$ and $\bar{\lambda}_{i}$ only differ by the constant $a$. The derivative $\psi_{\lambda_{i}}$ may be obtained explicitly by applying the following property of the hypergeometric function ${ }_{2} F_{1},[28]$ :

$$
\frac{\partial_{2} F_{1}(a, b, c, z)}{\partial z}=\frac{a b}{c}{ }_{2} F_{1}(a+1, b+1, c+1, z) .
$$

The material elasticity tensor $\mathbb{C}_{\text {tissue }}$ associated with $\mathbf{S}_{\text {tissue }}$ is given by

$$
\mathbb{C}_{\text {tissue }}=2 \frac{\partial \mathbf{S}_{\text {tissue }}}{\partial \mathbf{C}}=\mathbb{C}_{\text {vol }}+\mathbb{C}_{\text {matrix }}+\mathbb{C}_{\text {fibers }} \text {, }
$$

with

$$
\mathbb{C}_{\text {fibers }}=2 \sum_{i=1}^{N} \frac{\partial \mathbf{S}_{\text {fibers }}}{\partial \mathbf{C}}=\sum_{i=1}^{N}\left(\frac{\psi_{\lambda_{i} \lambda_{i}}}{\lambda_{i}^{2}}-\frac{\psi_{\lambda_{i}}}{\lambda_{i}^{3}}\right) \mathbf{A}_{i} \otimes \mathbf{A}_{i},
$$

where $\psi_{\lambda_{i} \lambda_{i}}=\partial^{2} \psi_{\text {fibers }}\left(\lambda_{i}\right) / \partial \lambda_{i}^{2}$. The tensor $\mathbb{C}_{\text {vol }}$ in (31) is given in [17], p. 254, and $\mathbb{C}_{\text {matrix }}$ reduces to the (fourth-order) zero tensor for the neo-Hookean model. Note the explicit appearance of the structure tensor $\mathbf{A}_{i}$ associated with each fiber family $i$ in the expressions for the stress and elasticity tensors.

The engineering (first Piola-Kirchhoff) stress tensor $\mathbf{P}$ then follows from $\mathbf{S}$ through the relation $\mathbf{P}=\mathbf{F S}$.

\subsection{Uniaxial extension test with loading along the fiber direction}

We consider now a fibrous tissue with only one collagen fiber family $(N=1)$ subject to stretch-controlled uniaxial extension along the fiber direction, as shown in Fig. 4(a). The tissue is stretched until individual collagen fibers fail, then it is unloaded and reloaded until complete tissue failure. In order to focus just on the fibers, in this example we omit the contribution due to the matrix material so that the parameter $c$ in $(26)_{1}$ is zero. In several fibrous tissues the matrix material is essentially an aqueous substance with negligible tensile stiffness in comparison with the collagen fibers, which explains the high compliance of these tissues in the low-stretch domain and justifies the above assumption.

The resulting relations between the engineering stress $P$ and the stretch $\bar{\lambda}$ for $m \leq \epsilon_{\max }$ and $m>\epsilon_{\max }$ are plotted in Figs. 4(b) and 4(c), respectively. These plots are independent of the parameter $a$, i.e. the value of $\bar{\ell}$ when $\sigma_{d}^{2}$ 


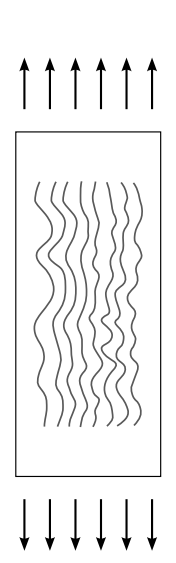

(a)

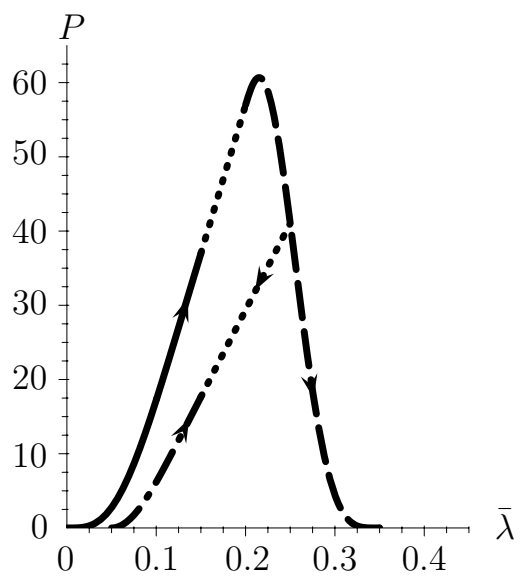

(b) $m \leq \epsilon_{\max }$

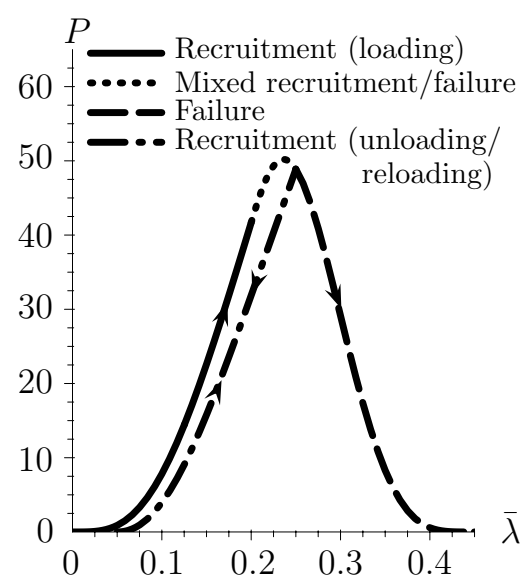

(c) $m>\epsilon_{\max }$

Fig. 4. (a) Schematic representation of the uniaxial extension test along the fiber direction; (b), (c) engineering stress $P$ (in MPa) versus stretch $\bar{\lambda}$ for loading and unloading/reloading paths for $m \leq \epsilon_{\max }$ and $m>\epsilon_{\max }$, respectively. Solid curves corresponds to the elastic collagen recruitment phase; dotted curves relate to the phase in which all collagen fibers are fully recruited, i.e. (b), and to the mixed recruitment/failure phase, i.e. (c); dashed curves correspond to the failure phase and dash-dotted curves to the recruitment phase in the unloading/reloading paths.

takes on its smallest value. The different $m$ values do not affect the shape of the Beta distribution or the cumulative strain energy required to stretch the tissue until complete failure. However, as can be seen by comparing the two plots in Fig. 4, the fibers are recruited more rapidly and may sustain higher stresses for $m \leq \epsilon_{\max }$, although they fail more abruptly; total fiber failure is reached at a smaller stretch value $\bar{\lambda}$ for the case $m \leq \epsilon_{\max }$. In addition, when full recruitment is achieved the linear stress-stretch relationship that describes the behavior of a single fiber is recovered, while this is not the case for $m>\epsilon_{\max }$.

Although $\psi_{\text {fibers }}$, and hence its derivatives, are defined piecewise, see (25), Figs. 4(b) and (c) feature smooth curves. In fact, $\psi_{\text {fibers }}$ is twice differentiable in the whole domain of the monotonically increasing loading paths, and also for the unloading/reloading paths except for the stretch $\bar{\lambda}$ at $b+\epsilon_{\max }=0.25$. This is due to the smoothness of the Beta distribution that describes the fiber morphology at the tissue level. Note, however, that special methods such as the arc-length method must be applied in a numerical simulation in order to handle the softening effect induced by the progressive failure of the fibers, which results in a negative definite tangent matrix. 


\subsubsection{Effect of the shape parameters $\eta$ and $\gamma$ on the stress-stretch behavior}

We consider the same loading protocol as above to study the effect of the shape parameters $\eta$ and $\gamma$ on the Beta distribution, and, therefore, on the final form of the strain energy $\psi_{\text {fibers }}$ and the resulting stress-stretch behavior.

Depending on the values of $m$ and $\epsilon_{\max }$, there are basically two different stressstretch behaviors for each pair of $(\eta, \gamma)$. Here we restrict our analysis to bellshaped Beta distributions $(\gamma>1$ and $\eta>1)$, and investigate two cases with equal shape parameters $(\gamma=\eta=2$ and $\gamma=\eta=8)$, which lead to symmetric Beta distributions, and two additional cases with $\gamma=2, \eta=8$ and $\gamma=8$, $\eta=2$. The results are plotted in Fig. 5 and organized as follows: the first column shows the Beta distribution over the normalized domain $\bar{\ell} / m$ for the given values of the shape parameters; the second and third columns show the related stress-stretch relations in terms of engineering stress $P$ and stretch $\bar{\lambda}$ for $m \leq \epsilon_{\max }$ and $m>\epsilon_{\max }$, respectively, where the unloading curves have been omitted. The patterns for the individual curves are as shown in Fig. 4. Except for $\gamma$ and $\eta$ all material parameters are taken from Table 1.

As expected, for the case $m \leq \epsilon_{\max }$ the linear stress-stretch relationship is recovered for the phase in which all fibers are recruited. For all displayed curves for $m \leq \epsilon_{\max }$ the maximum stress value is higher than for $m>\epsilon_{\max }$. Higher values for $\eta$ and $\gamma$ tend to make the bell of the Beta distribution narrower and higher, and to increase the maximum stress value (compare the first two rows in Fig. 5). For non-symmetric Beta distributions, i.e. the third and fourth rows in Fig. 5, the final shapes of the stress-stretch curves depend strongly on whether the distribution of $\bar{l}$ is shifted towards a lower or a higher value, i.e. whether or not $\gamma$ is lower or larger than $\eta$. For example, for $\gamma<\eta$, third row in Fig. 5, the Beta distribution is shifted towards the left, and the stress that the fibers can sustain decreases abruptly as soon as the first fiber fails (for $\bar{\lambda}>\epsilon_{\max }$ ). For $\gamma>\eta$, however, the Beta distribution is shifted towards the right, and the stress in the fibers is small when the (elastic) recruitment phase ends, but the stress continues to increase after the fibers start to fail.

\subsection{Pure shear test}

In this example we consider a fibrous tissue with two collagen fiber families $(N=2)$ subject to pure shear, as shown in Fig. 6. The fiber families are located in the $X-Y$ plane, and are symmetrically disposed with respect to the $X$ axis and described in terms of the angle $\theta$. The tissue is stretched in the $Y$-direction, while the length in the $X$-direction is kept constant. For that kinematics the matrix representation of the right Cauchy-Green tensor $\mathbf{C}$ has 

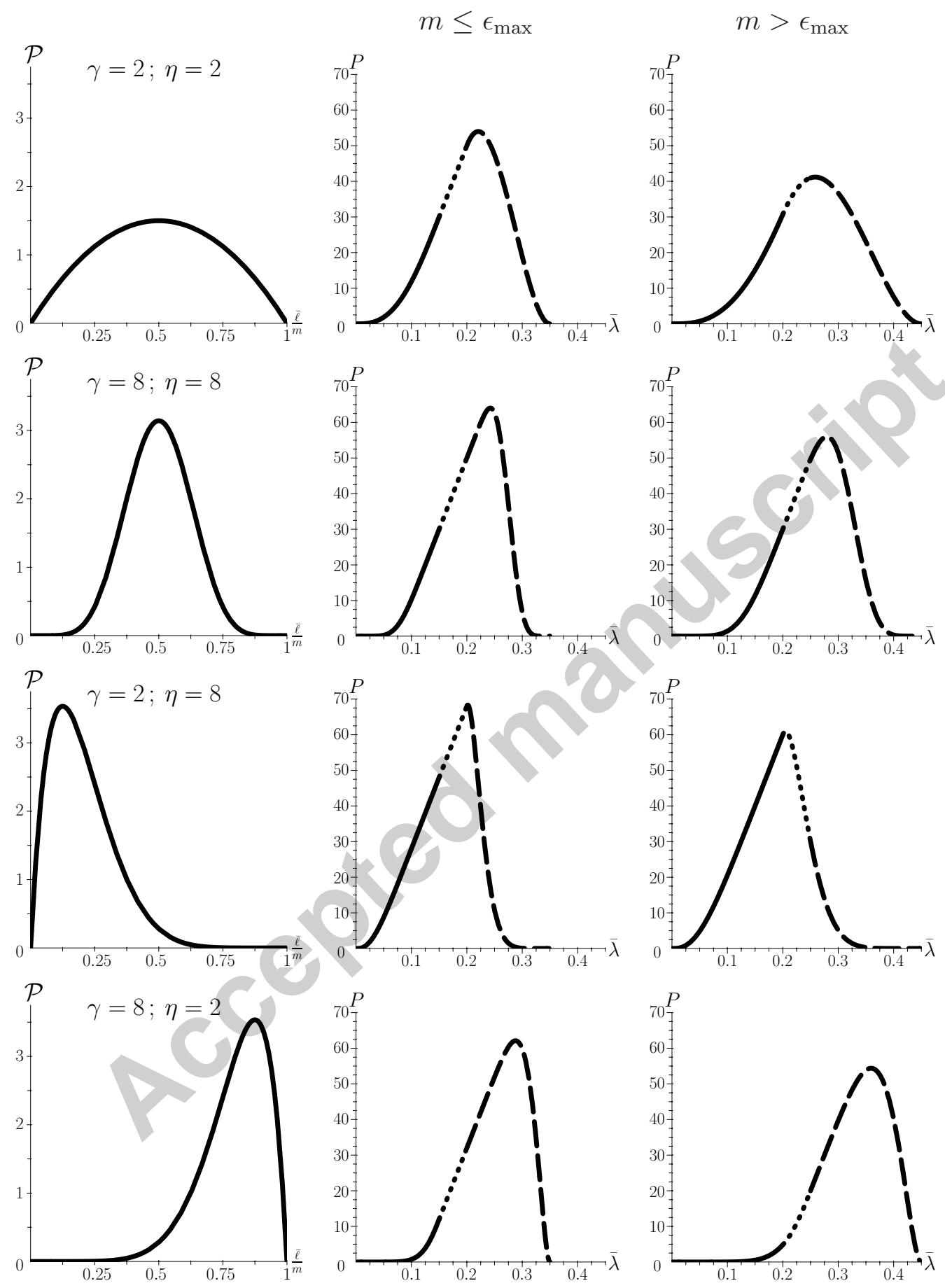

Fig. 5. Beta distributions $(\mathcal{P}$ versus $\bar{\ell} / m)$ and related stress-stretch relationships $(P$ versus $\bar{\lambda}$ ) for four sets of shape parameters $\eta$ and $\gamma$, and for the cases $m \leq \epsilon_{\max }$ and $m>\epsilon_{\max }$. Units for $P$ are given in MPa. The patterns for the individual curves are as in Fig. 4. 


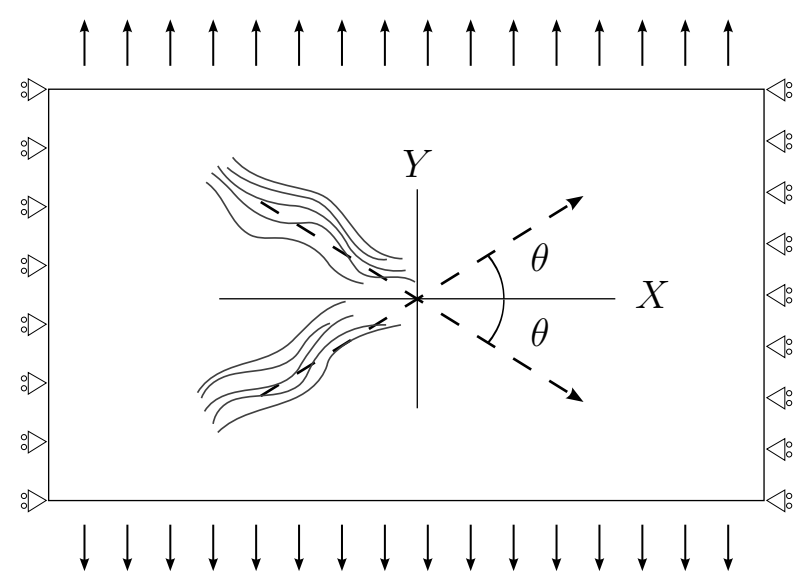

Fig. 6. Schematic representation of the pure shear test.

the form

$$
[\mathbf{C}]=\left[\begin{array}{ccc}
1 & 0 & 0 \\
0 & \lambda^{2} & 0 \\
0 & 0 & \lambda^{-2}
\end{array}\right],
$$

where $\lambda$ denotes the stretch in the $Y$-direction. According to the material and load symmetries, the stretch in the fibers is the same for both families. Thus, from (10) we find that

$$
\lambda_{i}=\left(\mathbf{C}: \mathbf{a}_{i_{0}} \otimes \mathbf{a}_{i_{0}}\right)^{1 / 2}=\left(\cos ^{2} \theta+\lambda^{2} \sin ^{2} \theta\right)^{1 / 2}, \quad i=1,2,
$$

where $\lambda_{1}=\lambda_{2}$. The material parameters are taken from Table 1 . For the matrix material a (neo-Hookean) parameter with value $c=15 \mathrm{kPa}$ was chosen for the model (26).

In Fig. 7 the engineering stress components $P_{x}$ and $P_{y}$ in the $X$ and $Y$ directions are plotted versus the stretch $\lambda$ for different fiber orientations $\theta$ $\left(90^{\circ}, 60^{\circ}, 50^{\circ}, 40^{\circ}, 30^{\circ}, 15^{\circ}\right)$, and for monotonic loading up to $\lambda=1.75$. Plots are shown for the two cases $m \leq \epsilon_{\max }$ and $m>\epsilon_{\max }$. No unloading paths are presented for clarity.

The case for which the fibers are located at $\theta=0^{\circ}$ (in the $X$-direction) is considered to be special because, according to (34), because the stretch $\lambda_{1}=\lambda_{2}=1$ in the fiber families is independent of the applied stretch $\lambda$. Hence, the stress contribution due to the fibers is zero. In the case of $\theta=90^{\circ}$, for which the fibers are located along the $Y$-direction, the stretch $\lambda_{1}=\lambda_{2}$ in the fiber families is equal to the applied stretch $\lambda$. Hence, the corresponding component of the structure tensor is zero and the stress contribution of the fibers in the $X$-direction vanishes. These apparently counterintuitive results are a consequence of the consideration that the collagen fibers are parallel and 

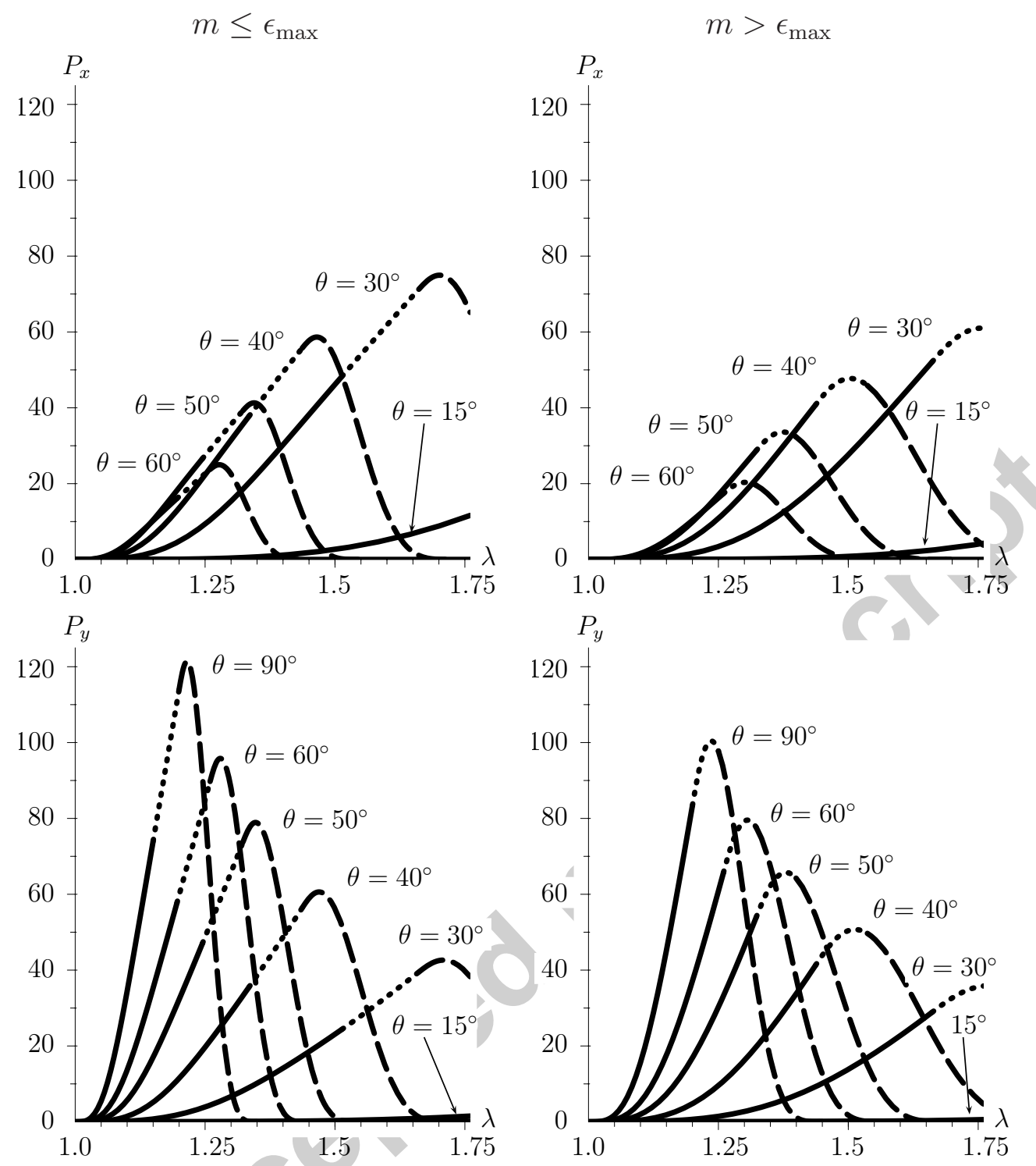

Fig. 7. Pure shear test for different fiber orientations and for the two cases $m \leq \epsilon_{\max }$ and $m>\epsilon_{\mathrm{max}}$ : engineering stress components $P_{x}, P_{y}$ (in $X$ and $Y$-directions) versus stretch $\lambda$ applied in the $Y$-direction, compare with Fig. 5. Units for the stresses are given in MPa. The patterns for the individual curves are as in Fig. 4.

decoupled from the matrix material.

For all fiber orientations higher stresses are reached for the case $m \leq \epsilon_{\max }$. The stress-stretch relationship is also linear in the fully recruited phase. As the fibers are increasingly aligned with the loading direction, the maximum stress $P_{y_{\max }}$ in the $Y$-direction increases, while complete tissue failure happens at smaller stretch values. Naturally, for the engineering stress in the $X$-direction, the maximum value $P_{x_{\max }}$ decreases with increasing alignment of the fiber family with the loading direction. This is due to the more progressive fiber 
recruitment at lower fiber angles.

For low values of $\theta$ complete tissue failure is not reached within the studied stretch range, as can be seen in the plots of Fig. 7. From the modeling point of view higher $\lambda$ values are not realistic since the matrix material would also be damaged. Finally, is is worth noting that the slopes of the stress-stretch curves change with the fiber orientation. For the $X$-direction, the slope increases with increasing $\theta$ until it reaches a maximum at about $45^{\circ}$, and then it decrease again. For the $Y$-direction, the slopes of the stress-stretch curves decreases monotonically with $\theta$.

\section{Discussion}

In this paper a new constitutive model for fibrous tissues that considers the randomly crimped morphology of the individual collagen fibers has been presented. On the basis of continuum mechanics the (macroscopic) constitutive model is formulated in terms of a few parameters, and it can capture material softening due to fiber failure. The collagen fiber is allowed to undulate randomly and its morphology may be described by a single parameter, which is measurable through micrographs using signal processing techniques. Although in the present model we have assumed that a single fiber may fail abruptly, the fibrous tissue fails progressively due to the different waviness of the fibers. This reflects our experimental observation when a fibrous tissue is stretched until rupture. This is also an important aspect for the numerical implementation in an implicit finite element code.

The proposed constitutive model is based on the idea that the energy required to move the fiber from the initially, crimped state to its fully extended state, is negligible in comparison with the energy required to stretch the fiber. Such an approach is relatively common in the literature and can be traced back to the work [35]. The linear stress-stretch relationship for the fiber was verified experimentally in [31], and also adopted here.

The basic approach proposed in this work is similar to that of others; see, e.g., $[19,23,21,36]$. However, the underlying tissue characterization is different since the statistical distribution can be measured [30]. This provides an 'objective' approach in the sense that the structure of different fibrous tissues, or regions within tissues, can be compared with each other without making assumptions about the collagen fiber length distribution. In the proposed approach the fibers must only be long enough with respect to their (random) wavelengths, which is the case for fibrous tissues. In addition, the proposed model enables the derivation of analytical expressions while capturing the complexity of the tissue behavior. For the implementation in a general code, it must be noted that the fibers remain one-dimensional entities irrespective of their spatial 
orientation. Therefore, according to Eq. $(34)_{1}$, their stress-stretch behavior is controlled by the scalar $\lambda$. The only difficulty is the implementation of the hypergeometric function. Although programming ${ }_{2} F_{1}(a, b, c, z)$ for general cases is not trivial, in our particular case (constant $a, b, c$ and real $z>1$ ) ${ }_{2} F_{1}$ is a single-valued function with no singularities. The hypergeometric function can either be applied directly from existing mathematical libraries, e.g. [37], or approximated by a quickly convergent series of exponential terms [28].

In the literature there are several other constitutive models for fibrous tissues available. Among these, a number of structural models have been proposed that characterize the fiber recruitment process by assuming that either the fiber length, [19,38,39,22,36], or the stretch at which the fibers engage [23,21] are statistically distributed. These models use unbounded distributions, which seem non-physiological for quantities such as the fiber length and the stretch. The choice of the Beta distribution overcomes this limitation. Fiber morphologies have also been incorporated into other constitutive models such as [40-42,24], although the characteristic waviness of the collagen fibers given therein has a pre-defined arrangement.

The proposed constitutive model assumes that the fibers are infinitesimally thin. It does not consider the volumetric fractions of collagenous and noncollagenous components. This fraction could essentially be considered by scaling the fiber elastic modulus $k$ and the neo-Hookean parameter $c$. The extent to which the fiber-matrix interaction contributes to the overall tissue stiffness is not known and, therefore, has not been considered. Similarly, the matrix is assumed to undergo finite deformations without damage. In addition, it has been assumed that the fibers do not interact with each other, which essentially implies that there is no fiber cross-linking. Moreover, the fibers do not interact with the matrix material. Images of human blood vessel samples obtained from, e.g., the outermost collagen-rich layer, taken through a microscope, reveal that collagen fibers are arranged very much in parallel, at least locally. This motivated the consideration of parallel fiber arrangement in the present approach. At other locations in a tissue region, however, the collagen fibers are aligned in a different direction. Hence, more globally, collagen fibers have distributed orientations which, in addition, need to be incorporated in a model, a step to be addressed in future.

\section{Acknowledgments}

Financial support for this study was partly provided by the Spanish Ministry of Education and Science under project numbers CICYT DPI2004-07410-C03 and HU2004-0009 ('Acciones Integradas'), and grant FP2000-5317 for F.C.; by the Spanish Ministry of Health through the National Network IM3 (Molecular 
and Multimodal Medical Imaging, 2003-2005); and by the Austrian Academy of Sciences with a doctoral grant for P.E. These supports are gratefully acknowledged.

\section{A Probability density function and mean of $\ell$}

\section{A.1 Probability density function $\mathcal{P}$ of $\ell$}

From eq. (2) we know that $\ell=\sqrt{2 d^{2}+1}$. Define now $r=2 d^{2}$, which is a random variable whose distribution is $\chi^{2}$ with $\sigma_{d}$, and thus

$$
\mathcal{P}(r)=\frac{1}{2 \sigma_{d}^{2}} \exp \left(-\frac{r}{2 \sigma_{d}^{2}}\right), \quad r \in[0, \infty)
$$

From the definitions of $\ell$ and $r$ it follows that $r=\ell^{2}-1$. Now for $\ell \geq 1$ we may derive the probability density function, and from (A.1) we find that

$$
\mathcal{P}(\ell)=\mathcal{P}[r(\ell)]\left|\frac{\partial r(\ell)}{\partial \ell}\right|=\frac{1}{2 \sigma_{d}^{2}} \exp \left(-\frac{\ell^{2}-1}{2 \sigma_{d}^{2}}\right)|2 \ell|,
$$

which is eq. (3), because $\ell$ is always positive.

\section{A.2 Mean $\bar{\ell}$ of $\ell$}

The mean $\bar{\ell}$ of $\ell$ is

$$
\begin{aligned}
\bar{\ell} & =\int_{\ell=1}^{\infty} \frac{\ell^{2}}{\sigma_{d}^{2}} \exp \left(-\frac{\ell^{2}-1}{2 \sigma_{d}^{2}}\right) \mathrm{d} \ell=\sigma_{d} \sqrt{2 \exp \left(1 / \sigma_{d}^{2}\right)} \int_{t=1 /\left(2 \sigma_{d}^{2}\right)}^{\infty} t^{1 / 2} \exp (-t) \mathrm{d} t \\
& =\sqrt{2 \sigma_{d}^{2} \exp \left(1 / \sigma_{d}^{2}\right)} \Gamma\left(\frac{3}{2}, \frac{1}{2 \sigma_{d}^{2}}\right)
\end{aligned}
$$

which is the desired result (5). In this derivation the variable change $t=$ $\ell^{2} /\left(2 \sigma_{d}^{2}\right)$ has been used, and the last equality follows from the definition of the incomplete Gamma function [28]. The same procedure can be used to calculate the variance of $\ell$. 


\section{References}

[1] N. Sasaki and S. Odajima. Stress-strain curve and Young's modulus of a collagen molecule as determined by the X-ray diffraction technique. J. Biomech., 29:655-658, 1996.

[2] S. Vesentini, C. F. Fitie, F. M. Montevecchi, and A. Redaelli. Molecular assessment of the elastic properties of collagen-like homotrimer sequences. Biomech. Model. Mechanobio., 3:224-234, 2005.

[3] Y. P. Kato, D. L. Christiansen, R. A. Hahn, S. J. Shieh, J. D. Goldstein, and F. H. Silver. Mechanical properties of collagen fibres: a comparison of reconstituted and rat tail tendon fibres. Biomaterials, 10:38-42, 1989.

[4] K. N. An, Y. L. Sun, and Z. P. Luo. Flexibility of type I collagen and mechanical property of connective tissue. Biorheology, 41:239-246, 2004.

[5] C. A. J. Schulze-Bauer, P. Regitnig, and G. A. Holzapfel. Mechanics of the human femoral adventitia including high-pressure response. Am. J. Physiol. Heart Circ. Physiol., 282:H2427-H2440, 2002.

[6] C. A. J. Schulze-Bauer, C. Mörth, and G. A. Holzapfel. Passive biaxial mechanical response of aged human iliac arteries. J. Biomech. Eng., 125:395406, 2003.

[7] G. A. Holzapfel, G. Sommer, C. T. Gasser, and P. Regitnig. Determination of the layer-specific mechanical properties of human coronary arteries with nonatherosclerotic intimal thickening, and related constitutive modelling. Am. J. Physiol. Heart Circ. Physiol., 289:H2048-2058, 2005.

[8] G. Sommer, T. C. Gasser, P. Regitnig, M. Auer, and G. A. Holzapfel. Dissection of the human aortic media: an experimental study. J. Biomech. Eng., 2007. in press.

[9] O. Kratky and G. Porod. Röntgenuntersuchung gelöster Fadenmoleküle. Recl. Trav. Chim. Pays-Bas., 68:1106-1123, 1949.

[10] J. F. Marko and E. D. Siggia. Stretching DNA. Macromolecules, 28:8759-8770, 1995.

[11] C. Bustamante, J. F. Marko, E. D. Siggia, and St. B. Smith. Entropic elasticity of $\lambda$-phage DNA. Science, 265:1599-1600, 1994.

[12] J. E. Adkins and R. S. Rivlin. Large elastic deformations of isotropic materials X. Reinforcement by inextensible cords. Philos. T. Roy. Soc. A, A248:201-223, 1955 .

[13] A. J. M. Spencer. Constitutive theory for strongly anisotropic solids. In A. J. M. Spencer, editor, Continuum Theory of the Mechanics of FibreReinforced Composites, pages 1-32. Springer-Verlag, Wien, 1984. CISM Courses and Lectures No. 282, International Centre for Mechanical Sciences. 
[14] G. A. Holzapfel, T. C. Gasser, and R. W. Ogden. A new constitutive framework for arterial wall mechanics and a comparative study of material models. $J$. Elasticity, 61:1-48, 2000.

[15] G. A. Holzapfel and R. W. Ogden, editors. Biomechanics of Soft Tissue in Cardiovascular Systems. Springer-Verlag, Wien - New York, 2003.

[16] G. A. Holzapfel and R. W. Ogden, editors. Mechanics of Biological Tissue. Springer-Verlag, Heidelberg, 2006.

[17] G. A. Holzapfel. Nonlinear Solid Mechanics. A Continuum Approach for Engineering. John Wiley \& Sons, Chichester, 2000.

[18] C. O. Horgan and G. Saccomandi. A description of arterial wall mechanics using limiting chain extensibility constitutive models. Biomech. Model. Mechanobio., 1:251-266, 2003.

[19] Y. Lanir. A structural theory for the homogeneous biaxial stress-strain relationships in flat collagenous tissues. J. Biomech., 12:423-436, 1979.

[20] Y. Lanir. Constitutive equations for fibrous connective tissues. J. Biomech., $16: 1-12,1983$.

[21] M. A. Zulliger, P. Fridez, K. Hayashi, and N. Stergiopulos. A strain energy function for arteries accounting for wall composition and structure. J. Biomech., 37:989-1000, 2004.

[22] F. L. Wuyts, V. J. Vanhuyse, G. J. Langewouters, W. F. Decraemer, E. R. Raman, and S. Buyle. Elastic properties of human aortas in relation to age and atherosclerosis: A structural model. Phys. Med. Biol., 40:1577-1597, 1995.

[23] C. Hurschler, B. Loitz-Ramage, and R. Vanderby Jr. A structurally based stress-stretch relationship for tendon and ligament. J. Biomech. Eng., 119:392399, 1997.

[24] A. D. Freed and T. C. Doehring. Elastic model for crimped collagen fibrils. J. Biomech. Eng., 127:587-593, 2005.

[25] A. D. Freed, D. R. Einstein, and I. Vesely. Invariant formulation for dispersed transverse isotropy in aortic heart valves: An efficient means for modeling fiber splay. Biomech. Model. Mechanobio., 4:100-117, 2005.

[26] F. Cacho. Constitutive models for fiber-reinforced soft biological tissues. PhD thesis, University of Zaragoza, Spain, 2006.

[27] T. C. Gasser, R. W. Ogden, and G. A. Holzapfel. Hyperelastic modelling of arterial layers with distributed collagen fibre orientations. J. R. Soc. Interface, 3:15-35, 2006.

[28] M. Abramowitz and I. A. Stegun. Handbook of Mathematical Functions With Formulas, Graphs, and Mathematical Tables. National Bureau of Standards, Applied Mathematics Series - 55, New York, 1972. 
[29] J. A. Trotter, F. A. Thurmond, and T. J. Koob. Molecular structure and functional morphology of echinoderm collagen fibrils. Conn. Tiss. Res., 275:451-458, 1994.

[30] P. J. Elbischger. Computer Vision Methods for the Automatic Analysis of Fibrous Structures in Biological Soft Tissues. PhD thesis, Graz University of Technology, 2005.

[31] N. Sasaki and S. Odajima. Elongation mechanism of collagen fibrils and forcestrain relations of tendon at each level of the structural hierarchy. J. Biomech., 29:1131-1136, 1996.

[32] G. A. Holzapfel and H. W. Weizsäcker. Biomechanical behavior of the arterial wall and its numerical characterization. Comp. Biol. Med., 28:377-392, 1998.

[33] R. W. Ogden and G. Saccomandi. Introducing mesoscopic information into constitutive equations for arterial walls. Biomech. Model. Mechanobio., 2007. DOI 10.1007/s10237-006-0064-8.

[34] J. J. Filliben and A. Heckert. NIST/SEMATECH e-Handbook of Statistical Methods. Chapter 1: Exploratory Data Analysis. 2006. http://www.itl.nist.gov/div898/handbook/index.htm.

[35] L. W. Lake and C. D. Armeniades. Structure-property relations of aortic tissue. Trans. Am. Soc. Artif. Intern. Organs, 18:202-209, 1972.

[36] J. F. Rodríguez, F. Cacho, J. A. Bea, and M. Doblaré. A stochastic-structurally based three dimensional finite-strain damage model for fibrous soft tissue. $J$. Mech. Phys. Solids, 54:864-886, 2006.

[37] M. Galassi, J. Davies, J. Theiler, B. Gough, G. Jungman, M. Booth, and F. Rossi. GNU Scientific Library Reference Manual - Revised Second Edition (v1.8). Network Theory Ltd., 2006.

[38] W. F. Decraemer, M. A. Maes, and V. J. Vanhuyse. An elastic stress-strain relation for soft biological tissues based on a structural model. J. Biomech., $13: 463-468,1980$.

[39] W. F. Decraemer, M. A. Maes, V. J. Vanhuyse, and P. Vanpeperstraete. A nonlinear viscoelastic constitutive equation for soft biological tissues, based upon a structural model. J. Biomech., 13:559-564, 1980.

[40] J. E. Bischoff, E. M. Arruda, and K. Grosh. Orthotropic hyperelasticity in terms of an arbitrary molecular chain model. J. Appl. Mech., 69:198-201, 2002.

[41] J. E. Bischoff, E. M. Arruda, and K. Grosh. A microstructurally based orthotropic hyperelastic constitutive law. J. Appl. Mech., 69:570-579, 2002.

[42] E. Kuhl, K. Garikipati, E. M. Arruda, and K. Grosh. Remodeling of biological tissue: Mechanically induced reorientation of a transversely isotropic chain network. J. Mech. Phys. Solids, 53:1552-1573, 2005. 